\title{
Tinnitus: clinical experience of the psychosomatic connection
}

This article was published in the following Dove Press journal:

Neuropsychiatric Disease and Treatment

10 February 2014

Number of times this article has been viewed

\author{
Massimo Salviati' \\ Francesco Saverio Bersani' \\ Samira Terlizzi' \\ Claudia Melcore' \\ Roberta Panico' \\ Graziella Francesca \\ Romano' \\ Guiseppe Valeriani' \\ Francesco Macrì' \\ Giancarlo Altissimi \\ Filippo Mazzei ${ }^{2}$ \\ Valeria Testugini ${ }^{2}$ \\ Luca Latini' \\ Roberto Delle Chiaie' \\ Massimo Biondi' \\ Giancarlo Cianfrone ${ }^{2}$ \\ 'Department of Neurology and \\ Psychiatry, Acute Psychiatric Ward \\ (Servizio Psichiatrico di Diagnosi e \\ Cura - SPDC), Sapienza University of \\ Rome, Rome, Italy, ${ }^{2}$ Department of \\ Sense Organs, Sapienza University of \\ Rome, Rome, Italy
}

Correspondence: Massimo Salviati Department of Neurology and Psychiatry, Sapienza University of Rome, Viale dell'Università $\mathrm{N}^{\circ} 30,00185$, Rome, Italy Tel +39064 4362895

Fax +39068079 418

Email massimo_salviati@yahoo.it
Background: The connection between psychopathology and tinnitus is complex and not adequately studied. The aim of this study is to investigate the relationship between tinnitus and psychiatric comorbidities from different points of view: categorical, dimensional, temperamental, and perceived stress level.

Methods: Two hundred and thirty-nine patients affected by tinnitus were recruited between January and October 2012. Patients underwent a preliminary battery of tests including the Tinnitus Handicap Inventory (THI), Symptom Check List (SCL90-R), Temperament and Character Inventory (TCI), and Stress-Related Vulnerability Scale (VRS), and eventually a full psychiatric evaluation.

Results: One hundred and fourteen patients ( $48 \%$ of the total sample) presented psychiatric comorbidity. Among these, a higher prevalence of depression, somatization, obsession, and anxiety was found. More than $41 \%$ of patients affected by decompensated tinnitus reported a family history of psychiatric disorders. Significant positive correlations between the psychopathological screening tools (SCL90-R and VRS) and THI were found. Patients affected by comorbid psychiatric disorder showed specific temperamental and characterial predispositions.

Conclusion: Psychiatric comorbidity in subjects affected by tinnitus is frequent. Stress can be considered as a factor leading to damage and dysfunction of the auditory apparatus. The vulnerability to neurotic disorders and the lack of coping capabilities can play a critical role in the clinical history of patients affected by severe tinnitus.

Keywords: tinnitus, psychosomatics, stress, psychopathological dimensions, personality

\section{Introduction}

Subjective tinnitus is an acoustic sensation perceived by the patient in one or both ears and/or in the head, which is not produced by any external source. Tinnitus can be experienced as a whistling, hissing, buzzing, or other sound, or as a pool of nondefined acoustic sensations; it can be continuous or intermittent. Patients affected by tinnitus usually experience the sound as originating in their head and heard through their ears. ${ }^{1}$ Tinnitus is a frequent symptom and it is estimated that approximately $15 \%-20 \%$ of the adult population usually experience it temporarily or permanently. ${ }^{2}$ Epidemiological studies report that $7 \%$ of the subjects affected by tinnitus require medical intervention and that the disorder is disabling in 5\% of these subjects, while it is considered a strong handicap in $1 \%-2 \%$ of the affected population, with severe consequences on quality of life. ${ }^{2}$ 
From an audiological point of view, the level of impairment caused by tinnitus is assessed by tests that estimate tinnitus-related distress. The most frequently used test is the Tinnitus Handicap Inventory questionnaire (THI), ${ }^{3}$ which describes five levels of intensity of the perceived impairment. Tinnitus noises usually become troublesome if patients focus their attention on them. ${ }^{4}$ In the medical literature, perception of tinnitus severity is correlated more closely with psychological and general health (such as pain or insomnia) factors than with audiometric parameters. ${ }^{5}$ The perception of tinnitus normally extinguishes in a short time through the "habituation" mechanism: superior brain centers activate thalamic filters to "switch off" the signal, often independently from the resolution of the dysfunction that originally generated the tinnitus. On the other hand, in the case of emotional reinforcements caused by fear, anxiety, or tension, the continued perception of tinnitus is supported by the limbic system, primarily by the amygdala; this establishes a vicious circuit which leads to the amplification (increased excitability) and the chronification (through neuronal plasticity mechanisms) of the signal. ${ }^{6}$ The validity of this model is supported by neurobiological studies that suggested that tinnitus is a phenomenon similar to the perception of chronic pain. ${ }^{7}$ This analogy is based on the concept that damages in peripheral structures, with long term subjective impairment, cause modifications in central signal processing mechanisms. ${ }^{8}$

Various studies showed that up to $77 \%$ of tinnitus sufferers may present psychiatric comorbidities. ${ }^{9,10}$ The outcomes of these studies also demonstrated that subjects with tinnitus show a lifetime prevalence of depression significantly higher than the general population. ${ }^{11}$ Some authors observed that in some cases psychiatric disorders could occur even a few years before the onset of the acoustic disorder. ${ }^{9,12}$ Belli et al showed that $27 \%$ of patients with tinnitus had at least one psychiatric diagnosis (anxiety disorders $28 \%$, somatoform and mood disorders $15 \%$, and personality disorders $3 \%$ ), versus $5.6 \%$ of control subjects. ${ }^{13}$ Anxious and depressive symptoms seem to be the most common complications of tinnitus. ${ }^{8,14}$ Folmer et al showed that tinnitus patients with concomitant depression reported the same level of tinnitus loudness of patients without a history of depression, but they had higher ratings of tinnitus severity. ${ }^{15}$ The same authors reported in a different work that tinnitus severity was positively correlated with quantitative measures of anxiety and depression. ${ }^{16}$ Moreover, psychosomatic studies identified some personality characteristics of subjects experiencing tinnitus, such as high level of alexithymia, ${ }^{17}$ elevation of the neurotic triad (hysteria, depression, and hypochondria; Hy-D-Hs) in the Minnesota Multiphasic Personality Inventory (MMPI) test, ${ }^{9}$ high levels of harm avoidance (HA) on the Tridimensional Personality Questionnaire (TPQ) test, ${ }^{18}$ neuroticism and lower levels of extraversion, ${ }^{19,20}$ withdrawal, and emotional isolation. $^{21}$

In regard to the relationship with stress, while most studies are focused on the role of tinnitus as a "stressor", 22 other papers describe how some stressful events can be identified prior to the emergence of the symptom. Also, a directly proportional relationship is identifiable between stress levels and intensity of perceived somatic distress. ${ }^{23}$ Perception of tinnitus can considerably vary in relation to coping capabilities and high stress levels ${ }^{24}$ and, on the other hand, abnormal physiological stress reactions might play a role in the onset, maintenance, and exacerbation of tinnitus symptoms. ${ }^{25,26}$ The results of experimentally induced biological stress reactions are partially inconsistent but indicate a different cortisol reaction pattern in tinnitus patients compared to controls..$^{27,28}$

The relationship between tinnitus and stress can also involve a connection between inner ear sensitivity and neuroendocrinological, immune, and toxic variations linked to stress activation. ${ }^{29}$ The role of psychosomatics and stress reactions in the development of tinnitus is corroborated by the neurophysiological model proposed by Jastreboff and Hazell. ${ }^{30}$ According to this model tinnitus becomes chronic and decompensated as a consequence of malfunctioning circuiting in a complex neural network that involves sensory, limbic, and autonomic components, ${ }^{23}$ independently from the causes that generated tinnitus; alterations in the same neural network have been described in subjects affected by emotional and anxiety disorders, ${ }^{31}$ and it is known from animal studies that psychopharmacological treatment produces a "normalization effect" on the functionality of these areas. ${ }^{32}$ Positron emission tomography (PET) and functional magnetic resonance imaging (fMRI) studies also confirmed parallel activations in auditory, reticular, limbic and autonomic regions in subjects affected by tinnitus. ${ }^{23}$

In conclusion, the connection between psychopathology, tinnitus and treatment is complex and, perhaps not sufficiently investigated with adequate methodologies. The aim of this study is to deeply investigate the relationship between tinnitus and psychiatric comorbidities from different points of view: categorical, dimensional, temperamental, and perceived stress levels. 


\section{Materials and methods}

The evaluation was carried out on patients referred to Associazione Italiana per la Ricerca sulla Sordità (Italian Association for Deafness Research; [AIRS]) Tinnitus Center of the Department of Sense Organs of Policlinico Umberto I University Hospital in Rome, according to the following criteria: categorical (Diagnostic and Statistical Manual of Mental Disorders Fourth edition Text Revision [DSM IV-TR] diagnosis), dimensional (The Symptom Checklist - 90 - Revised [SCL90-R]), temperamental and characterial (Temperament and Character Inventory [TCI]) and perceived stress level (Stress-Related Vulnerability Scale [VRS]). Furthermore, anamnestic data were collected about premorbid psychopathology and previous exposure to stressful events (according to the Paykel scale of stressful life events) in the period before the onset of tinnitus..$^{33,34}$

Between January and October 2012, 239 patients were enrolled in the study: 151 males and 88 females, with a mean age \pm standard deviation of $52.07 \pm 14.04$ years (range, 13-81). The duration of tinnitus was not considered as inclusion criteria.

The following assessment operations were carried out on the overall sample:

1. Psychopathological screening: patients were informed on tinnitus neurophysiological model and treatment; a battery of self-completion tests including THI, SCL90$\mathrm{R}$, and VRS was delivered to the subjects in order to divide the sample in two subgroups: patients presenting high probability of a psychiatric comorbidity ("high- $\psi$ ") and patients presenting low probability of a psychiatric comorbidity ("low- $\psi$ "); ${ }^{35}$

2. Personological evaluation: TCI vas delivered to the whole sample;

3. Psychiatric evaluation: the high- $\psi$ subgroup was examined by a psychiatrist who confirmed ( $\psi$ subgroup) or disconfirmed ( $\psi$ - subgroup) the presence of psychiatric comorbidities, formulated the categorical diagnosis, and collected anamnestic data;

4. Intergroup confrontation: patients who were diagnosed with psychiatric comorbidity $(\psi)$ were compared to the non-psychiatric group ( $\psi-$ ) from the dimensional viewpoint; moreover, the average scores of personological variables were compared to normative values;

5. Intragroup correlations: the association between quantitative psychopathological variables and the intensity of the perceived tinnitus-related distress (THI) was investigated on both the groups of patients ( $\psi$ and $\psi-$ ).

\section{Psychopathological screening and personological evaluation}

Three tests (THI, SCL90-R, and VRS) were used as a screening method in order to evaluate the necessity of a psychiatric examination to assess psychiatric comorbidities.

As described in the introduction, the THI had been used in the past as a sensitive screening tool; it describes five levels of intensity of the perceived impairment on the basis of the scores obtained: "very mild" (0-16); "mild" (18-36); "moderate" (38-56); "severe" (58-76); and "catastrophic" (78-100). Scores higher than 36 are considered as indicative of a condition of "decompensation" which requires psychological or psychiatric assessment. ${ }^{36}$ However, in previous studies this quantitative indication (THI>36) has been found to be poorly specific for the detection of "decompensated tinnnitus" (in our previous research the same cut-off showed a sensitiviy of $86.8 \%$ and a specificity of $59.1 \%)^{22,35}$

The SCL90-R test is one of the few self-administration psychometric tests that can be used both in dimensional evaluation and as a screener for psychopathological aspects also on non-psychiatric subjects. Some studies found a correlation between THI scores and SCL90-R scores. ${ }^{22}$ The SCL90-R includes 90 items, which constitute nine dimensions (I - somatization; II - obsessive-compulsive; III - interpersonal sensitivity; IV - depression; V - anxiety; VI - hostility; VII - phobic anxiety; VIII - paranoid ideation; IX - psychoticism). The SCL90-R test also provides an index, the General Symptomatic Index (GSI), which discriminates subjects at high risk of psychiatric disorder if higher than $0.567 .{ }^{37}$

The VRS is a swift evaluation tool of perceived stress. It consists of 13 items with an answering grading between 0 and 3 . On the basis of the global scores obtained, the stress level of the subject is categorized as follows: scores between 0 and 19 "mild stress"; scores between 20 and 26 "moderate stress"; and scores between 27 and 39 "severe stress". ${ }^{38}$ Subjects presenting a moderate or severe level of stress were normally advised to get further and more specific examinations in order to detect a possible presence of a psychopathological disorder. The score $>17$ was arbitrarily taken as a cut-off for pathological stress according to our previous study. ${ }^{35}$

The presence of two "positive" tests out of three for the of psychiatric co-morbidity $(\mathrm{THI}>36$; GSI $>0.567$; VRS $>17$ ) allowed us to identify the sample of patients of the "high- $\psi$ " group who would undergo psychiatric evaluation (according to this method, in a previous study of our research team a sensitivity of $91.2 \%$ and a specificity of $79.5 \%$ were found). ${ }^{35}$ 
The presence of dysfunctional temperaments and characters was evaluated through the TCI score. The $\mathrm{TCI}^{39}$ consists of a self-evaluation questionnaire, which, in its most complete version, includes 240 items with true/false answers. The test explores four temperament dimensions (novelty-seeking, harm avoidance, reward dependence, and persistence) and three character dimensions (self-directedness, cooperativeness, and self-transcendence). A brief description of these personological traits is reported in Table 1. The presence of personological patterns of predisposition to tinnitus development was investigated by comparing the TCI profiles of the sample (both on the whole and split in the two sub-samples $\psi$ and $\psi-$ ) with the population-based normative values.

\section{Psychiatric evaluation}

Patients positive to the psychopathological screening ("high- $\psi$ ") were visited by a senior psychiatrist who confirmed or disconfirmed the presence of psychiatric comorbidities, formulated a categorical diagnosis in accordance with the DSM IV-TR criteria and investigated the presence of stressful events in the 6 months preceding tinnitus onset, familial psychiatric disorders, and possible earlier psychopathological episodes.

Patients who were part of the "high- $\psi$ " group after the psychopathological screening but were negative to the psychiatric evaluation were shifted to the $\psi$ - group.

\section{Statistical analysis}

Comparisons between quantitative variables were carried out using an analysis of variance (ANOVA) test. A correlation between quantitative variables was performed using the Pearson correlation test. Significance

Table I TCl: description of temperament and character traits

Temperament traits

- Novelty seeking: tendency towards exploration, intense activation, and excitability

- Harm avoidance: tendency towards avoidance of aversive stimuli

- Reward dependence: tendency toward an intense response to social reward

- Persistence: perseverance in spite of fatigue and frustration

Character traits

- Self-directedness: strong-will individuals, capable of controlling, regulating and adapting their behavior in order to best reach their goals

- Cooperativeness: individuals who are tolerant, empathic, compassionate, and supportive of others

- Self-transcendence: relevant to individuals who conceive themselves as integral parts of the universe as a whole, a "unitary conscience" in which everything is part of a whole; this perspective can be described as acceptance, identification, or spiritual unity with nature and its origins

Note: Data from Tarsitani et al..$^{38}$ levels of $P<0.01$ were arbitrarily chosen to indicate significance.

\section{Results}

Of the total of 239 initial patients, 121 patients were in the high- $\psi$ group and, among these, 114 patients ( $48 \%$ of the total sample) were in the $\psi$ group, ie, they were diagnosed with a psychiatric disorder. The $\psi$ group included 64 males and 50 females, with an average age \pm standard deviation of 51.21 \pm 14.57 (range 13-81 years; Table 2).

In the $\psi$ group, 23 patients $(20.17 \%)$ satisfied the criteria for a diagnosis of non specific anxiety disorder, 19 patients $(16.67 \%)$ of generalized anxiety disorder, 14 patients $(12.28 \%)$ of adaptive disorder, eleven subjects of dysthymia (9.64\%), eleven patients $(9.64 \%)$ of somatization disorder, ten patients of cyclothymic disorder (8.77\%), eight patients of recurrent depressive disorder (7.02\%), eight patients $(7.02 \%)$ of major depressive disorder, and seven patients (6.14\%) of obsessive-compulsive disorder. The final three patients (each representing $0.88 \%$ of the total sample) were diagnosed as affected by bipolar disorder, conversion disorder, and panic disorder.

Two-thirds $(66.66 \%)$ of $\psi$ patients $(\mathrm{N}=76)$ had a stressful event (according to the Paykel stressful events list) during the 6 months preceding onset of tinnitus. Among these, ten patients had stressful events involving the "work" field, five involving the "education" field, 12 involving the "finances" field, 16 involving the "health field", three involving the "bereavement" field, two involving the "change of residence" field, seven involving the "courtship-engagement-cohabitation" field, four involving the "legal matters" field, 14 involving the "family and social relations" field and three involving the "marriage" field.

More than half of $\psi$ patients $(51.75 \%$; $=59)$ presented psychiatric disorders prior to the onset of tinnitus.

Fifty $\psi$ patients (43.86\%) had already used psychotropic drugs before undergoing the psychopathological tests of this study.

Forty-seven $\psi$ patients $(41.22 \%)$ affected by decompensated tinnitus reported a family history of psychiatric disorders.

Table 2 Frequencies of psychiatric disorders among sexes

\begin{tabular}{|c|c|c|c|c|}
\hline$\Psi$ or $\Psi-$ & Male & Female & Total & $\chi^{2}$ Test \\
\hline$\Psi$ & $64(42.38 \%)$ & $50(56.82 \%)$ & II 4 (47.70\%) & 4 4.643; degrees \\
\hline$\Psi-$ & 87 (57.62\%) & 38 (43.18\%) & 125 (52.30\%) & $\begin{array}{l}\text { of freedom: I; } \\
P=0.03 \mathrm{I}\end{array}$ \\
\hline Total & $|5|$ & 88 & 239 & \\
\hline
\end{tabular}

Notes: $\Psi$, patients with psychiatric comorbidity; $\Psi-$, patients without psychiatric comorbidity. 
The average scores of the screening evaluation showed low to moderate stress levels (VRS $=17.95 \pm 7.48$ ), high likelihood of a psychiatric disorder (GSI $=0.76 \pm 0.49$ ), and moderate level of tinnitus-related distress $(\mathrm{THI}=46.98 \pm 27.83$; Table 3).

From a dimensional viewpoint, a higher prevalence of depression, somatization, obsession, and anxiety compared to the other dimensions investigated was found (Table 4).

Subjects with psychiatric comorbidity $(\psi)$ showed significantly higher scores for all psychopathological dimensions compared to patients without psychiatric comorbidity $(\psi-)$, (Table 5).

The global sample showed erratic mean values within the range of normality, while the values of SD (self-directedness) and $\mathrm{C}$ (cooperativeness) characters were slightly higher than average (Table 6).

By comparing personological variables between subjects with $(\psi)$ and without $(\psi-)$ psychiatric comorbidity, significant differences were found for what concerns HA (significantly higher in patients with psychiatric comorbidity) and SD (significantly lower in the same group of patients; Table 7).

Investigating the correlation between psychometric variables and THI, significant linear correlations were found between the psychopathological screening tools (GSI and VRS) and the THI (Table 8); all psychopathological dimensions of the SCL-90R test are directly correlated with the screening measures (Table 9); the only personological dimensions which correlated with all the other variables (level of perceived stress, gravity of the psychopathology, and intensity of "decompensation" of tinnitus) were the HA (direct correlation) and the SD (inverse correlation) (Table 10).

\section{Discussion}

This study is a preliminary investigation of the psychopathological components related to tinnitus and it is part of a larger project entailing a joint work between psychiatrists and audiologists in the multidisciplinary therapeutic management of the disorder. To our knowledge, this is the

Table 3 Screening tests

\begin{tabular}{lll}
\hline Test & Mean & Standard deviation \\
\hline VRS & 17.95 & 7.48 \\
GSI & 0.76 & 0.49 \\
THI & 46.98 & 27.83 \\
\hline
\end{tabular}

Abbreviations: GSI, General Symptomatic Index; THI, Tinnitus Handicap Inventory; VRS, Stress-Related Vulnerability Scale.
Table 4 Psychopathological dimensions of the whole sample of patients (SCL 90-R)

\begin{tabular}{lll}
\hline SCL90-R dimensions & Mean & Standard deviation \\
\hline Somatization & 10.85 & 7.21 \\
Obsessive-compulsive & 10.03 & 7.11 \\
Sensitivity & 5.30 & 5.40 \\
Depression & 12.40 & 9.02 \\
Anxiety & 8.51 & 6.20 \\
Hostility & 3.93 & 3.08 \\
Phobic anxiety & 2.36 & 3.07 \\
Paranoid ideation & 4.20 & 3.85 \\
Psychoticism & 4.94 & 5.23 \\
\hline
\end{tabular}

Abbreviation: SCL90-R, The Symptom Checklist - 90 - Revised.

first study evaluating stress, personality, psychopathological dimensions, and psychiatric diagnostic categories in the same sample of tinnitus-affected patients.

In our sample, men presented tinnitus more frequently than women (151 males and 88 females, a ratio of 1.72), in agreement with what was reported by Pilgram et al. ${ }^{40}$ Pilgram et al, in fact, reported in an epidemiological study that tinnitus is more frequent in males than in females. ${ }^{40}$ However, the results of our study cannot be extended to the whole population at the moment. The average age of the sample (52.07 years) suggests that tinnitus is primarily associated with aging, although the age of the sample ranged between 13 and 81 . In a recent epidemiological study it has been found that the prevalence of tinnitus in the population was higher in 55-84 year old people than in younger participants (14.4\% versus $9.0 \%$, respectively; $P<0.001)$ with an increasing, statistically significant, linear trend in prevalence by age group for men, women, and both sexes; however, the authors of the paper also reported data showing a decrease of prevalence of tinnitus after the 7 th and 8 th decades of life. ${ }^{41}$

In the present study, age did not correlate with THI score, with the intensity of perceived distress nor with any of the psychopathological dimensions investigated. The link between aging and tinnitus seems to be based on impaired hearing status ${ }^{42}$ and hearing loss seems to be the strongest risk factor associated with tinnitus. ${ }^{41}$ Longitudinal data are needed to understand the impact of aging on the development of tinnitus and on the degree of related suffering.

In our sample, psychiatric comorbidity was frequent $(48 \%)$, as already reported in scientific literature; the group of $\psi$ subjects showed a balanced male to female ratio (1.28) and the percentage of patients with psychiatric comorbidity was higher in women than in men $(56.82 \%$ versus $42.38 \%$, respectively). These data are consistent with the recent finding that women seem to be more disturbed by tinnitus 
Table 5 Psychopathological dimensions: psychiatric $(\Psi)$ versus non-psychiatric $(\Psi-)$ groups

\begin{tabular}{|c|c|c|c|c|c|c|}
\hline \multirow[t]{2}{*}{ SCL 90-R } & \multicolumn{2}{|l|}{$\Psi-$} & \multicolumn{2}{|l|}{$\Psi$} & \multicolumn{2}{|c|}{ Comparisons (ANOVA) } \\
\hline & Mean & Standard deviation & Mean & Standard deviation & $\Psi / \Psi-$ & $P$-value \\
\hline Somatization & 6.99 & 5.024 & 14.72 & 7.544 & 2.105866 & $<0.001$ \\
\hline Obsessive-compulsive & 5.33 & 4.362 & 13.80 & 7.601 & 2.589118 & $<0.001$ \\
\hline Sensitivity & 2.91 & 2.923 & 7.96 & 6.037 & 2.735395 & $<0.001$ \\
\hline Depression & 6.00 & 4.572 & 18.02 & 8.255 & 3.003333 & $<0.001$ \\
\hline Anxiety & 4.31 & 3.074 & 12.34 & 6.173 & 2.863109 & $<0.001$ \\
\hline Hostility & 2.44 & 2.098 & 5.40 & 3.402 & 2.213115 & $<0.001$ \\
\hline Phobic anxiety & 1.10 & 1.842 & 3.54 & 3.464 & 3.218182 & $<0.001$ \\
\hline Paranoid ideation & 2.12 & 2.219 & 5.85 & 3.895 & 2.759434 & $<0.001$ \\
\hline Psychoticism & 1.88 & 2.522 & 7.44 & 5.214 & 3.957447 & $<0.001$ \\
\hline
\end{tabular}

Notes: $\Psi$, patients with psychiatric comorbidity; $\Psi$-, patients without psychiatric comorbidity.

Abbreviations: ANOVA, analysis of variance; SCL 90-R, The Symptom Checklist - 90 - Revised.

and perceive it as more stressful than men. ${ }^{43}$ No statistically significant differences between women and men were found regarding THI, SCL90-R, or VRS scores.

Tinnitus-related distress was moderate $(\mathrm{THI}=46.98 \pm 27.83)$ in the enrolled patients; this impairment directly correlated with high levels of psychopathology and stress, as also reported in previous studies. ${ }^{914-16,22,35}$

The findings of the study suggest that the psychopathological factors related to tinnitus should not be solely seen as a complication or decompensation of tinnitus: $66 \%$ of $\psi$ patients reported to have had stressful events prior to tinnitus onset; the intensity of the distress correlated with the level of perceived stress (correlation between VRS and THI); 51.75\% of patients $(\mathrm{N}=59)$ who underwent psychiatric evaluation already showed psychiatric disorders before tinnitus onset; $43.86 \%(\mathrm{~N}=50)$ of patients presenting psychiatric disorders had already used psychotropic drugs before the diagnosis; $41.22 \%$ of $\psi$ subjects $(\mathrm{N}=47)$ reported a family history of psychiatric disorders.

In the light of these observations, it is still not possible to postulate the presence of a psychopathologically determined vulnerability to tinnitus onset. Although the

Table $6 \mathrm{TCl}$ : temperament and character dimensions of the whole sample of patients

\begin{tabular}{lll}
\hline & Mean & Normal range \\
\hline HA & 18.089 & $11.9-23.34$ \\
RD & 14.10 & $10.88-21.34$ \\
NS & 16.91 & $13.6-26.68$ \\
P & 4.37 & $2.72-5.336$ \\
SD & 30.60 & $14.96-29.35$ \\
C & 29.96 & $14.28-28.01$ \\
ST & 14.40 & $11.22-22.01$ \\
\hline
\end{tabular}

Abbreviations: C, cooperativeness; HA, harm avoidance; NS, novelty seeking; $\mathrm{P}$, persistence; $\mathrm{RD}$, reward dependence; $\mathrm{SD}$, self directiveness; $\mathrm{ST}$, self-transcendence; $\mathrm{TCl}$, Temperament and Character Inventory. presence of a specific dimensional psychopathological profile (prevalence of dimensions such as depression, somatization, obsession, and anxiety) allows to categorize the sample by symptomatology, it does not provide a clear cut result for the presence of a tinnitus susceptibility pattern.

On the other hand, the study on temperament and character provides the datum of a personological predisposition for the development of "severe tinnitus". As a matter of fact, correlation tests showed that there is there is a relationship between severity of tinnitus and HA (direct correlation) and $\mathrm{SD}$ (inverse correlation). These results support the hypothesis of a predisposition to the development of "severe" tinnitus, and not to the development of tinnitus in general. More specifically, the HA trait was identified as a predisposing factor for the development of disorders of the neurotic area (anxiety, emotional, and obsessive disorders in particular), while low levels of SD are typical of subjects showing weak willpower and reduced stress coping capabilities ${ }^{39}$ (similar

Table $7 \mathrm{TCl}$ : psychiatric $(\Psi)$ versus non-psychiatric $(\Psi-)$ group

\begin{tabular}{lllll}
\hline $\begin{array}{llll}\text { TCl comparisons } \\
\text { (ANOVA) }\end{array}$ & $\Psi-$ & & $\Psi$ & $P$-value \\
\cline { 2 - 2 } & Mean & & Mean & \\
\hline HA & 15.12 & & 20.89 & $<0.001$ \\
RD & 14.18 & & 14.03 & NS \\
NS & 16.85 & 16.97 & NS \\
P & 4.62 & & 4.13 & NS \\
SD & 33.39 & 27.97 & $<0.001$ \\
C & 30.91 & 29.07 & NS \\
ST & 14.06 & 14.71 & NS \\
\hline
\end{tabular}

Notes: $\Psi$, patients with psychiatric comorbidity; $\Psi-$, patients without psychiatric comorbidity.

Abbreviations: ANOVA, analysis of variance; C, cooperativeness; HA, harm avoidance; NS, novelty seeking; P, persistence; RD, reward dependence; SD, self directiveness; ST, self-transcendence; $\mathrm{TCl}$, Temperament and Character Inventory. 
Table 8 Correlation between screening measures in the whole sample of patients

\begin{tabular}{|c|c|c|c|}
\hline Correlations & VRS & GSI & THI \\
\hline \multicolumn{4}{|l|}{ VRS } \\
\hline Pearson correlation & I & $0.800(* *)$ & $0.636(* *)$ \\
\hline Significance (2-tailed) & & $P<0.00$ I & $P<0.00$ I \\
\hline \multicolumn{4}{|l|}{ GSI } \\
\hline Pearson correlation & $0.800(* *)$ & I & $0.553(* *)$ \\
\hline Significance (2-tailed) & $P<0.001$ & & $P<0.001$ \\
\hline \multicolumn{4}{|l|}{ THI } \\
\hline Pearson correlation & $0.636(* *)$ & $0.553(* *)$ & 1 \\
\hline Significance (2-tailed) & $P<0.001$ & $P<0.001$ & \\
\hline
\end{tabular}

Note: $* * P<0.01$.

Abbreviations: GSI, General Symptomatic Index; THI, Tinnitus Handicap Inventory; VRS, Stress-Related Vulnerability Scale.

findings have already been described in medical literature). ${ }^{18}$ The constitutional difficulty to cope with stress accounts for the correlation between tinnitus-related distress perception and stress. According to the results of the research, decompensated tinnitus can be considered as the ill-fated meeting

Table 9 Correlation between psychopathological dimensions and screening in the whole sample of patients

\begin{tabular}{|c|c|c|c|}
\hline $\begin{array}{l}\text { Correlation } \\
\text { SCL-90 R dimensions }\end{array}$ & VRS & GSI & THI \\
\hline \multicolumn{4}{|l|}{ Somatization } \\
\hline Pearson correlation & $0.588(* *)$ & $0.760(* *)$ & $0.421(* *)$ \\
\hline Significance (2-tailed) & $P<0.001$ & $P<0.001$ & $P<0.001$ \\
\hline \multicolumn{4}{|l|}{ Obsessive-compulsive } \\
\hline Pearson correlation & $0.703(* *)$ & $0.869(* *)$ & $0.482(* *)$ \\
\hline Significance (2-tailed) & $P<0.001$ & $P<0.001$ & $P<0.001$ \\
\hline \multicolumn{4}{|l|}{ Sensitivity } \\
\hline Pearson correlation & $0.576(* *)$ & $0.796(* *)$ & $0.370(* *)$ \\
\hline Significance (2-tailed) & $P<0.001$ & $P<0.001$ & $P<0.001$ \\
\hline \multicolumn{4}{|l|}{ Depression } \\
\hline Pearson correlation & $0.793(* *)$ & $0.935(* *)$ & $0.565(* *)$ \\
\hline Significance (2-tailed) & $P<0.001$ & $P<0.001$ & $P<0.001$ \\
\hline \multicolumn{4}{|l|}{ Anxiety } \\
\hline Pearson correlation & 0.726 (**) & $0.872(* *)$ & $0.536(* *)$ \\
\hline Significance (2-tailed) & $P<0.001$ & $P<0.00 I$ & $P<0.001$ \\
\hline \multicolumn{4}{|l|}{ Hostility } \\
\hline Pearson correlation & $0.600(* *)$ & $0.689(* *)$ & $0.380(* *)$ \\
\hline Significance (2-tailed) & $P<0.001$ & $P<0.001$ & $P<0.001$ \\
\hline \multicolumn{4}{|l|}{ Phobic anxiety } \\
\hline Pearson correlation & $0.443(* *)$ & $0.597(* *)$ & $0.398(* *)$ \\
\hline Significance (2-tailed) & $P<0.00$ I & $P<0.00 I$ & $P<0.001$ \\
\hline \multicolumn{4}{|l|}{ Paranoid ideation } \\
\hline Pearson correlation & $0.562(* *)$ & $0.738(* *)$ & $0.366(* *)$ \\
\hline Significance (2-tailed) & $P<0.00$ I & $P<0.00 I$ & $P<0.001$ \\
\hline \multicolumn{4}{|l|}{ Psychoticism } \\
\hline Pearson correlation & $0.614(* *)$ & $0.85 \mathrm{I}(* *)$ & $0.425(* *)$ \\
\hline Significance (2-tailed) & $P<0.001$ & $P<0.001$ & $P<0.00$ I \\
\hline
\end{tabular}

Note: $* * P<0.01$.

Abbreviations: SCL-90 R, Symptom Check List; GSI, General Symptomatic Index; THI, Tinnitus Handicap Inventory; VRS, Stress-Related Vulnerability Scale.
Table 10 Correlation between $\mathrm{TCl}$ dimension and screening measures in the whole sample of patients

\begin{tabular}{|c|c|c|c|}
\hline $\mathrm{TCl}$ correlation & VRS & GSI & THI \\
\hline \multicolumn{4}{|l|}{$\mathrm{HA}$} \\
\hline Pearson correlation & $0.567(* *)$ & $0.624(* *)$ & $0.340(* *)$ \\
\hline Significance (2-tailed) & $P<0.00 I$ & $P<0.001$ & $P<0.001$ \\
\hline \multicolumn{4}{|l|}{ RD } \\
\hline Pearson correlation & 0.015 & 0.121 & 0.014 \\
\hline Significance (2-tailed) & 0.866 & 0.162 & 0.875 \\
\hline \multicolumn{4}{|l|}{ NS } \\
\hline Pearson correlation & 0.045 & 0.145 & -0.012 \\
\hline Significance (2-tailed) & 0.602 & 0.093 & 0.895 \\
\hline \multicolumn{4}{|l|}{$P$} \\
\hline Pearson correlation & -0.144 & -0.088 & -0.034 \\
\hline Significance (2-tailed) & 0.095 & 0.31 & 0.697 \\
\hline \multicolumn{4}{|l|}{ SD } \\
\hline Pearson correlation & $-0.56 \mathrm{I}(* *)$ & $-0.646(* *)$ & $-0.245(* *)$ \\
\hline Significance (2-tailed) & $P<0.00$ I & $P<0.00$ I & 0.004 \\
\hline \multicolumn{4}{|l|}{ C } \\
\hline Pearson correlation & $-0.216(*)$ & -0.109 & -0.087 \\
\hline Significance (2-tailed) & 0.012 & 0.206 & 0.319 \\
\hline \multicolumn{4}{|l|}{ ST } \\
\hline Pearson correlation & 0.054 & 0.233 (**) $^{* *}$ & 0.11 \\
\hline Significance (2-tailed) & 0.529 & 0.006 & 0.207 \\
\hline
\end{tabular}

Notes: $* P<0.05 ; * * p<0.01$.

Abbreviations: C, cooperativeness; GSI, General Symptomatic Index; HA, harm avoidance; NS, novelty seeking; P, persistence; RD, reward dependence; SD, self directiveness; ST, self-transcendence; TCl, Temperament and Character Inventory; THI, Tinnitus Handicap Inventory; VRS, Stress-Related Vulnerability Scale.

between frequent pathological phenomena: psychiatric disorders and tinnitus.

According to the stress vulnerability model proposed by Zubin and Spring in 1977, the severity of tinnitus may be explained as a particular vulnerability to the distressing impact of the tinnitus sound. ${ }^{44}$

One of the limitations of the present study is that the psychiatric evaluation was limited to subjects who underwent an initial psychopathological screening which showed a very low frequency of false positives (7) while, since the "low $\psi$ " subjects were not evaluated, it is not possible to assess the presence of false negatives in the rest of the sample. Another limitation of the study is the lack of categorization of the sample in relation to the characteristics of tinnitus (duration and type) and the audiometric patterns of patients.

\section{Conclusion}

Psychiatric comorbidity in subjects affected by tinnitus is frequent, even if this study showed that the relationship between psychopathology and tinnitus is not linear.

With respect to the pathogenetic model of tinnitus, the following observations can be made: 
1. Tinnitus onset originates at various levels in the acoustic district; stress can be included among the causes of damage or dysfunction of the delicate auditory apparatus; ${ }^{29}$

2. the presence of a predisposition to neurotic disorders and the lack of coping capabilities could play critical roles in the clinical history of patients affected by severe tinnitus (psychiatric diathesis; previous psychiatric disorders; limbic dysfunction not allowing the activation of the habituation mechanism; development of adaptation disorders to tinnitus-generated stress; reaching unbearable levels of distress and low compliance to rehab treatments);

3. chronification of tinnitus depends on pathological plasticity mechanisms in the tonotopical organization of the cortex;

4. severity of tinnitus seems to be linked to temperamental and characterial vulnerabilities, psychopathological dimensions, and stress levels.

The present work represents a starting point for a project on multidisciplinary therapeutic approaches to tinnitus.

\section{Acknowledgments}

This research was supported by AIRS (Associazione Italiana per la Ricerca sulla Sordità - Italian Association for the Research on Deafness).

\section{Disclosure}

The authors report no conflicts of interest in this work.

\section{References}

1. Cianfrone G, Passi S. Acufeni e iperacusia. Il trattamento riabilitativo. Audiologia - Newsletter. 2004;8:1-20.

2. Heller AJ. Classification and epidemiology of tinnitus. Otolaryngol Clin North Am. 2003;36(2):239-248.

3. Newman CW, Jacobson GP, Spitzer JB. Development of the Tinnitus Handicap Inventory. Arch Otolaryngol Head Neck Surg. 1996;122(2): 143-148.

4. Hallam RS, Jakes SC, Hinchcliffe R. Cognitive variables in tinnitus annoyance. Br J Clin Psychol. 1988;27(Pt 3):213-222.

5. Baskill, JL, Coles, RR, Lutman, ME, Axelsson, A. Tinnitus severity grading: longitudinal studies. In: Fourth International Tinnitus. Seminar, Bord, France 1991:457-460.

6. Georgiewa P, Klapp BF, Fischer F, et al. An integrative model of developing tinnitus based on recent neurobiological findings. Med Hypotheses. 2006;66(3):592-600.

7. Møller AR. Similarities between chronic pain and tinnitus. Am J Otol. 1997;18(5):577-585.

8. Salvi RJ, Henderson D, Hamernik R, Ahroon WA. Neural correlates of sensorineural hearing loss. Ear Hear. 1983;4(3):115-129.

9. Marciano E, Carrabba L, Giannini P, et al. Psychiatric comorbidity in a population of outpatients affected by tinnitus. Int J Audiol. 2003; 42(1):4-9.

10. Konzag TA, Rübler D, Bandemer-Greulich U, Frommer J, Fikentscher E. [Psychological comorbidity in subacute and chronic tinnitus outpatients]. Z Psychosom Med Psychother. 2005;51(3):247-260. German.
11. Harrop-Griffiths J, Katon W, Dobie R, Sakai C, Russo J. Chronic tinnitus: association with psychiatric diagnoses. J Psychosom Res. 1987;31(5):613-621.

12. Unterrainer J, Greimel KV, Leibetseder M, Koller T. Experiencing tinnitus: which factors are important for perceived severity of the symptom? Int Tinnitus J. 2003;9(2):130-133.

13. Belli S, Belli H, Bahcebasi T, Ozcetin A, Alpay E, Ertem U. Assessment of psychopathological aspects and psychiatric comorbidities in patients affected by tinnitus. Eur Arch Otorhinolaryngol. 2008;265(3): 279-285.

14. Stobik C, Weber RK, Münte TF, Walter M, Frommer J. Evidence of psychosomatic influences in compensated and decompensated tinnitus. Int J Audiol. 2005;44(6):370-378.

15. Folmer RL, Griest SE, Meikle MB, Martin WH. Tinnitus severity, loudness, and depression. Otolaryngol Head Neck Surg. 1999;121(1): 48-51.

16. Folmer RL, Griest SE, Martin WH. Obsessive-compulsiveness in a population of tinnitus patients. Int Tinnitus J. 2008;14(2):127-130.

17. Salonen J, Johansson R, Joukamaa M. Alexithymia, depression and tinnitus in elderly people. Gen Hosp Psychiatry. 2007;29(5):431-435.

18. Russo J, Katon W, Sullivan M, Clark M, Buchwald D. Severity of somatization and its relationship to psychiatric disorders and personality. Psychosomatics. 1994;35(6):546-556.

19. Zachariae R, Mirz F, Johansen LV, Andersen SE, Bjerring P, Pedersen CB. Reliability and validity of a Danish adaptation of the Tinnitus Handicap Inventory. Scand Audiol. 2000;29(1):37-43.

20. Rizzardo R, Savastano M, Maron MB, Mangialaio M, Salvadori L. Psychological distress in patients with tinnitus. $J$ Otolaryngol. 1998;27(1):21-25.

21. Bayar N, Oğuztürk O, Koç C. Minnesota Multiphasic Personality Inventory profile of patients with subjective tinnitus. $J$ Otolaryngol. 2002;31(5):317-322.

22. Bauch CD, Lynn SG, Williams DE, Mellon MW, Weaver AL. Tinnitus impact: three different measurement tools. J Am Acad Audiol. 2003;14(4):181-187.

23. Georgiewa P, Klapp BF, Fischer F, et al. An integrative model of developing tinnitus based on recent neurobiological findings. Med Hypotheses. 2006;66(3):592-600.

24. Schneider WR, Hilk A, Franzen U. [Social support, disability, coping with stress and personality markers in patients with subjective chronic aural tinnitus and a clinical control group]. HNO. 1994;42(1):22-27. German.

25. Horner KC. The emotional ear in stress. Neurosci Biobehav Rev. 2003;27(5):437-446.

26. Mazurek B, Stöver T, Haupt H, et al. [The significance of stress: its role in the auditory system and the pathogenesis of tinnitus]. HNO. 2010;58(2):162-172. German.

27. Hébert S, Paiement P, Lupien SJ. A physiological correlate for the intolerance to both internal and external sounds. Hear Res. 2004;190(1-2):1-9.

28. Hébert S, Lupien SJ. The sound of stress: blunted cortisol reactivity to psychosocial stress in tinnitus sufferers. Neurosci Lett. 2007;411(2): $138-142$.

29. Horner KC. The emotional ear in stress. Neurosci Biobehav Rev. 2003;27(5):437-446.

30. Hazell JW, Jastreboff PJ. Tinnitus. I: Auditory mechanisms: a model for tinnitus and hearing impairment. J Otolaryngol. 1990;19(1):1-5.

31. Anand A, Shekhar A. Brain imaging studies in mood and anxiety disorders: special emphasis on the amygdala. Ann N Y Acad Sci. 2003;985:370-388.

32. John F, Cryana JF, Valentino RJ. Assessing substrates underlying the behavioral effects of antidepressants using the modified rat forced swimming test. Neurosci Biobehav Rev. 2005;29:547-569.

33. Paykel ES, Prusoff BA, Uhlenhuth EH. Scaling of life events. Arch Gen Psychiatry. 1971;25(4):340-347.

34. Fava GA, Osti RMA. Versione Italiana della scala di Paykel per gli eventi stressanti. Florence: Organizzazioni Speciali, 1981. 
35. Salviati M, Macrì F, Terlizzi S, et al. The Tinnitus Handicap Inventory as a screening test for psychiatric comorbidity in patients with tinnitus. Psychosomatics. 2012;54(3):248-256.

36. Aksoy S, Firat Y, Alpar R. The Tinnitus Handicap Inventory: a study of validity and reliability. Int Tinnitus J. 2007;13(2):94-98.

37. Schauenburg H, Strack M. Measuring psychotherapeutic change with the symptom checklist SCL 90R. Psychother Psychosom. 1999;68(4): 199-206.

38. Tarsitani L, Biondi M. [Development and validation of the VRS, a rating scale for rapid stress assessment] Sviluppo e validazione della scala VRS (Valutazione Rapida dello Stress). Med Psicosom. 1999;3:163-177. Italian.

39. Cloninger CR, Przybeck TR, Svrakic DM, Wetzel RD. The Temperament and Character Inventory (TCI): A Guide to its Development and Use. St Louis: Washington University; 1994.
40. Pilgramm M, Rychlick R, Lebisch $\mathrm{H}$ et al. Tinnitus in the Federal Republic of Germany: A representative epidemiological study; in Hazell J (ed): Proceedings of the $\mathrm{VI}^{\text {th }}$ International Tinnitus Seminar. Cambridge, The Tinnitus and Hyperacusis Center, 1999, pp 64-67.

41. Nondahl DM, Cruickshanks KJ, Huang GH, et al. Tinnitus and its risk factors in the Beaver Dam offspring study. Int J Audiol. 2011;50(5): 313-320.

42. Martines F, Bentivegna D, Martines E, Sciacca V, Martinciglio G. Characteristics of tinnitus with or without hearing loss: clinical observations in Sicilian tinnitus patients. Auris Nasus Larynx. 2010; 37(6):685-693.

43. Seydel C, Haupt H, Olze H, Szczepek AJ, Mazurek B. Gender and chronic tinnitus: differences in tinnitus-related distress depend on age and duration of tinnitus. Ear Hear. 2013;34(5):661-672.

44. Zubin J, Spring B. Vulnerability - a new view of schizophrenia. J Abnorm Psychol. 1977;86(2):103-126.

\section{Publish your work in this journal}

Neuropsychiatric Disease and Treatment is an international, peerreviewed journal of clinical therapeutics and pharmacology focusing on concise rapid reporting of clinical or pre-clinical studies on a range of neuropsychiatric and neurological disorders. This journal is indexed on PubMed Central, the 'PsycINFO' database and CAS.
The manuscript management system is completely online and includes a very quick and fair peer-review system, which is all easy to use. Visit http://www.dovepress.com/testimonials.php to read real quotes from published authors.

Submit your manuscript here: http://www.dovepress.com/neuropsychiatric-disease-and-treatment-journal 